\title{
Label-Free Quantitative Analysis Using LC/MS
}

\author{
Atsumu Hirabayashi \\ Hitachi, Ltd., \\ Japan
}

\section{Introduction}

Quantitative analysis using liquid chromatography/mass spectrometry (LC/MS) is widespread, especially in drug metabolism and pharmacokinetics studies, and in many laboratories label-free LC/MS analyses are carried out, i.e., without using isotope labeling techniques. However, even if the analytical method is well validated, an unexpected change in matrix concentrations in biological samples may cause matrix effects such as ion suppression or ion enhancement. When ion suppression occurs, for example, the ionization efficiency of an analyte molecule decreases, and the ion intensity of the analyte decreases from the expected intensity (Tang et al., 2004; Buhrman et al., 1996). Then the linear relationship between the sample amount and the ion intensity is lost, as shown in Figure 1.

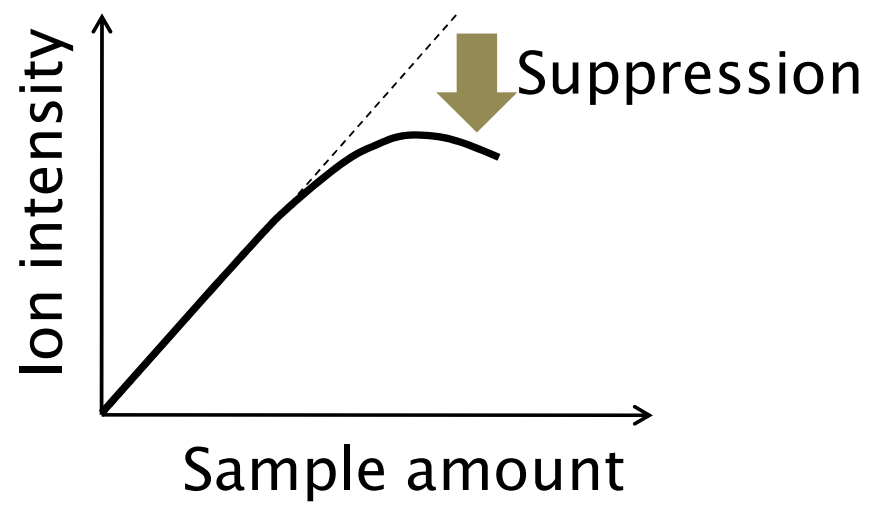

Fig. 1. When ion suppression occurs, the linear relationship between the ion intensity and the sample amount is lost, as shown by an arrow, and thus, label-free quantitative analysis becomes difficult.

The effects of ion suppression can be overcome by using stable-isotope labeling techniques such as ${ }^{12} \mathrm{C} /{ }^{13} \mathrm{C}$ and ${ }^{14} \mathrm{~N} /{ }^{15} \mathrm{~N}$ labeling, since an isotope-labeled molecule, used as an internal standard, exhibits chemical properties or effects of ion suppression almost identical to those of the unlabeled one. Eventually, though, label-free LC/MS analyses have to be carried out when we cannot employ isotope labeling techniques, which are rather laborious and expensive. 
Ion suppression occurs in LC/MS interfaces such as electrospray ionization (ESI) and atmospheric pressure chemical ionization (APCI), which are used to analyze less volatile and volatile molecules, respectively. Because ESI is the more widely used technique, ion suppression in ESI is mainly described here, although that in APCI is also discussed later.

Because the effect of ion suppression depends on the chemical properties of the analyte molecule in ESI, it is difficult to correct for decreased intensity, so quantitative analysis becomes difficult. The potential occurrence of ion suppression can be reduced by desalting and fractionating the sample, reducing the sample volume, and the effect of ion suppression can be reduced by using a structural analog. Nevertheless, ion suppression may occur, and correcting for the decreased intensity remains difficult. Several sample preparation protocols for specific analytes have been proposed to reduce the effect of ion suppression by using internal standards (Matuszewski et al., 1998; Bonfiglio et al., 1999), but they are not widely used in label-free LC/MS.

We have developed a simple technique for detecting potential ion suppression in ESI (Hirabayashi et al., 2007, 2009). In this technique, a specific concentration of a probe molecule, which is sensitive to the occurrence of ion suppression, is added to an LC mobile phase, and the intensity of the protonated probe molecule is monitored. When ion suppression occurs, the intensity of the protonated probe is expected to decrease more than those of other protonated molecules, $[\mathrm{M}+\mathrm{H}]^{+}$.

\section{Ion formation from a charged droplet in ESI}

In this section, a brief explanation for why ion suppression occurs in ESI is presented, based on ion formation mechanisms of less volatile analyte molecules than solvent molecules, before explaining the technique for detecting potential ion suppression.

In ESI and other spray ionizations, an LC effluent in a capillary is sprayed from the capillary tip. Then, charged droplets are formed, from which gaseous ions are produced. Figure 2 shows a schematic view of a positively charged droplet. The droplet initially produced has a diameter of the order of $1 \mu \mathrm{m}$, and the diameter decreases as the solvent molecules evaporate. In the droplet, positive ions are concentrated near the inside of the droplet surface because of a Coulomb repulsive force. As solvent molecules evaporate, the gaseous ions are produced from the liquid-phase ions inside the droplet surface through ion evaporation (Iribarne \& Thomson, 1976; de la Mora, 2000) or charged residue mechanisms (Dole et al., 1968). Therefore, most ions analyzed in a mass spectrometer originate from the liquid-phase ones near the inside of the charged droplet surface. In the charged droplet, the liquid-phase chemistry, ignoring the effect of the surface, is not necessarily valid but is a good approximation (Hirabayashi 1993).

Ion suppression occurs in the ionization processes when a component eluted from an LC column affects the ionization of coeluted analytes. In the droplet surface, the analyte molecules are charged in accordance with their chemical properties when the number of charges is much higher than that of analyte molecules. Then the ionization efficiency for each analyte molecule remains constant. Under these conditions, label-free quantitative analysis is readily performed. On the contrary, when the number of charges in the droplet is comparable to, or less than, that of analyte molecules, charge competition occurs among the analyte molecules. Because the decrease in the number of charges can be regarded as an 
increase in $\mathrm{pH}$, the protonation for acidic molecules, for example, is likely to be reduced to a greater extent than that for neutral and basic ones. Of these molecules, those weakly dissociated near the surface tend to lose their charge in the competition, and their ionization efficiencies decrease, leading to ion suppression. Thus, in molecules with low ionization efficiencies, the ionization efficiencies tend to decrease further when ion suppression occurs. In contrast, when the number of charges in the droplet increases, the ionization efficiencies of some molecules increase. This leads to ion enhancement. The ionization efficiencies of molecules with low ionization efficiencies are expected to increase when ion enhancement occurs.

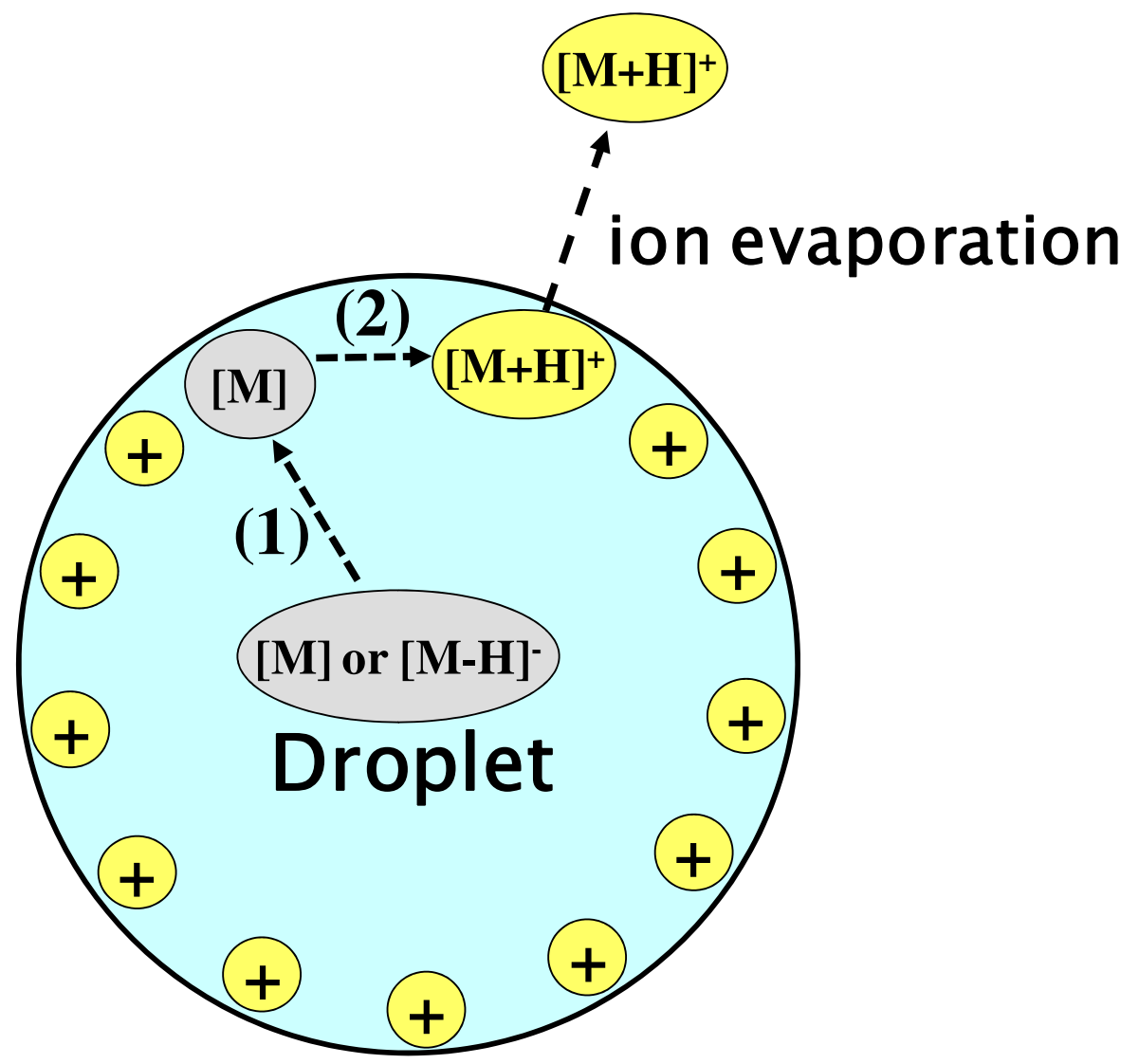

Fig. 2. Cross-sectional view of a positively charged droplet. The ionization processes of acidic molecules with low ionization efficiencies are shown: most of the molecules are deprotonated and concentrated in the central region of the droplet, but some do not undergo deprotonation because of their dissociation equilibrium. These neutral molecules with a low surface accessibility mostly remain in the droplet, but only a small part of the molecules can reach the droplet surface (1). Then, the protonated molecules may form near the surface (2). Gaseous ions are formed from the liquid-phase ions inside the droplet surface by ion evaporation or charge residue mechanisms. 


\section{Concept for ion suppression detection}

In the following we consider the ionization processes of molecules with low ionization efficiencies in a charge droplet. In the droplet, the neutral or negatively charged molecules remain deep, as shown in Fig. 2. To be protonated, these molecules have to access the surface. Then, near the inside of the droplet surface, they are protonated in accordance with their isoelectric point, or dissociation constant. Therefore, the major factors determining the ionization efficiency are (1) surface accessibility (or hydrophobicity) and (2) isoelectric point (or dissociation constant) of the analyte molecule. Hydrophobic and basic molecules are expected to be insensitive to ion suppression, and their ionization efficiencies are rather high. On the contrary, the ionization efficiencies of quite hydrophilic and acidic molecules are much lower than those of the above molecules. However, these molecules would be quite sensitive to the occurrence of ion suppression and can potentially be used as probes for detecting ion suppression in the analysis of positive ions (protonated molecules).

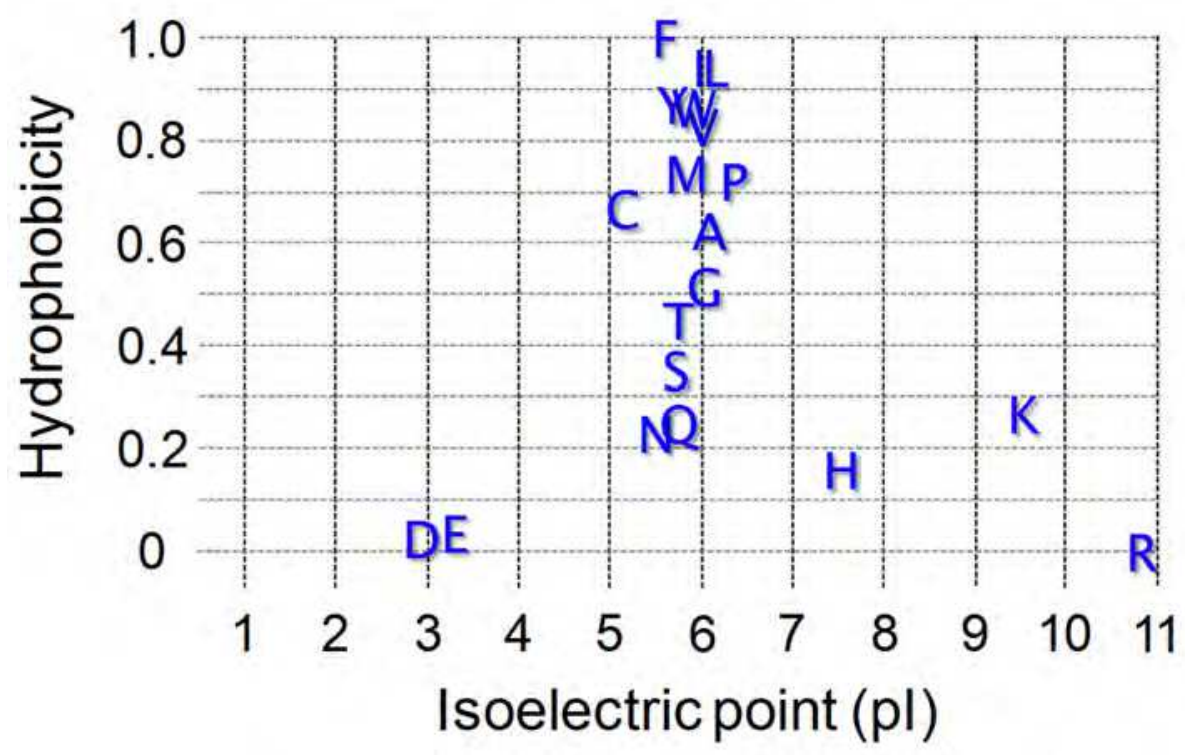

Fig. 3. Hydrophobicity (Black \& Mould, 1991) and isoelectric point (Linde, 1995) for 20 amino acids.

In developing the probe, a convenient technique is to synthesize a quite hydrophilic and acidic peptide, which has an amino-acid sequence including a hydrophilic and neutral amino acid as well as an acidic one, as the probe for detecting ion suppression. Figure 3 compares 20 amino acids in terms of their hydrophobicity and isoelectric points (pI). Among the amino acids, serine $(\mathrm{S})$, asparagine $(\mathrm{N})$, and glutamine $(\mathrm{Q})$ are very hydrophilic and neutral. On the other hand, aspartic acid (D) and glutamic acid (E) are very acidic, with a $\mathrm{pK}_{\mathrm{R}}$ of 3.65 and 4.25, respectively (Linde, 1995a), where $\mathrm{pK} \mathrm{K}_{\mathrm{R}}$ is the $\mathrm{pK}$ for the side chain of the amino acid. Then, as a probe for ion-suppression detection, we synthesized a peptide, DSSSSS, the isoelectric point of which is calculated to be 3.80 (Gasteiger et al., 2005). This 
probe is so acidic that no multiply protonated molecule of the probe is detected; only singly protonated ones can be detected at $\mathrm{m} / \mathrm{z} 569.3$ in a mass spectrometer. The molecular weight of the probe can be modified by altering the number of hydrophilic and neutral amino acids such as S. As mentioned above, the ionization efficiency of the probe is relatively low, whereas the probe concentration in the LC mobile phase should preferably be low, so as not to cause ion suppression. More acidic molecules than DSSSSS, for example, DDSSSS or DDDSSS with respective isoelectric points of 3.56 and 3.42, would be more sensitive to the occurrence of ion suppression, and could also be used as the probes. However, because of their higher acidities, their concentration should be higher than that of DSSSSS to be detected clearly in a mass spectrometer. Since the probe is rather insensitive to the occurrence of ion suppression of more acidic molecules than the probe, it is difficult to detect potential ion suppression for such acidic molecules if they are there. Note that the probe should be used to detect potential ion suppression for protonated molecules, not for cations and cationized molecules such as $[\mathrm{M}]^{+}$and $[\mathrm{M}+\mathrm{Na}]^{+}$.

For a probe used in negative-ion analysis, on the other hand, quite hydrophilic and basic peptides should be synthesized by using a very basic amino acid of $\mathrm{R}$ or $\mathrm{K}$ with a $\mathrm{pK} \mathrm{K}_{\mathrm{R}}$ of 12.48 and 10.53, respectively (Linde, 1995) and the very hydrophilic ones. Then ion suppression of ions with the form of $[\mathrm{M}-\mathrm{H}]-$ can be detected by monitoring the intensity of the deprotonated molecule of the probe.

In the following, the usability of the probe is examined. In most LC/MS analyses, the $\mathrm{pH}$ of the mobile phase ranges from 2 to 6 , and the concentration of the organic solvent such as acetonitrile and methanol is below $90 \%$. Figure 4 (a) plots the intensity of the protonated probe as a function of the acetonitrile concentration of the mobile phase under several $\mathrm{pH}$ conditions. The intensity appears to be almost independent of the mobile-phase $\mathrm{pH}$. On the other hand, the intensity increases with an increase in the acetonitrile concentration. The increase in the ion intensity (or the ion formation efficiency of the probe) can be ascribed to the enhanced solvent evaporation of the charged droplets, since the surface accessibility (or hydrophobicity) of the probe has been confirmed to be almost independent of the organic solvent concentration by comparing the probe with a much more hydrophobic peptide of FDFSF (Hirabayashi, 2009). Furthermore, the number of charges in the droplet is likely to be almost independent of the organic solvent concentration. This is because the ion current, which is the current for all of the ions and charged droplets produced by ESI, is almost unchanged, as shown in Fig. 4 (b), and this trend is independent of the organic solvent such as acetonitrile and methanol. Thus, the probe is expected to be much less surface-accessible and quite acidic under the conditions used in LC/MS analyses.

\section{Detection of ion suppression}

As mentioned earlier, the effect of ion suppression depends on the sample amount. Therefore, different sample amounts of fractionated human plasma, as a typically crude sample, were analyzed with a nanoLC/TOF-MS system to detect the occurrence of ion suppression. An aqueous solution of the probe is added to the LC mobile phase at a gradient mixer-pump unit of the nanoLC system just before being introduced into a separation column. Then, a linear gradient of acetonitrile concentration from 7 to $50 \%$ is run at a flow rate of $50 \mathrm{~nL} / \mathrm{min}$. Since the probe is very hydrophilic, it can pass through the reverse-phase separation column without adsorption when the organic solvent concentration of the LC 
mobile phase is above $4 \%$. Then the protonated probe is detected in the mass spectrometer during the LC/MS analysis.
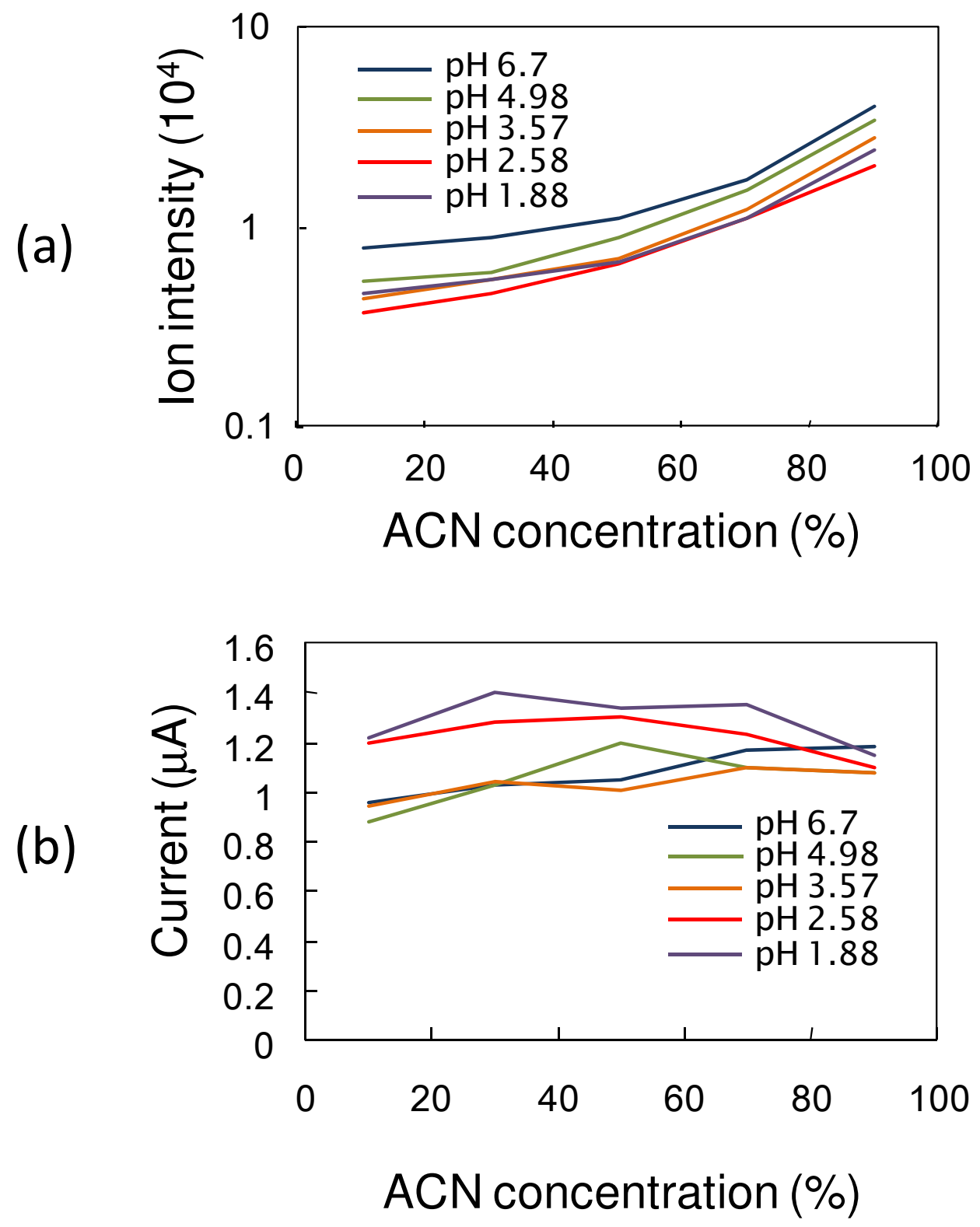

Fig. 4. (a) Intensity of the protonated probe molecule and (b) current for all the ions and charged droplets produced by ESI as a function of the acetonitrile (ACN) concentration of the mobile phase under several $\mathrm{pH}$ conditions. 

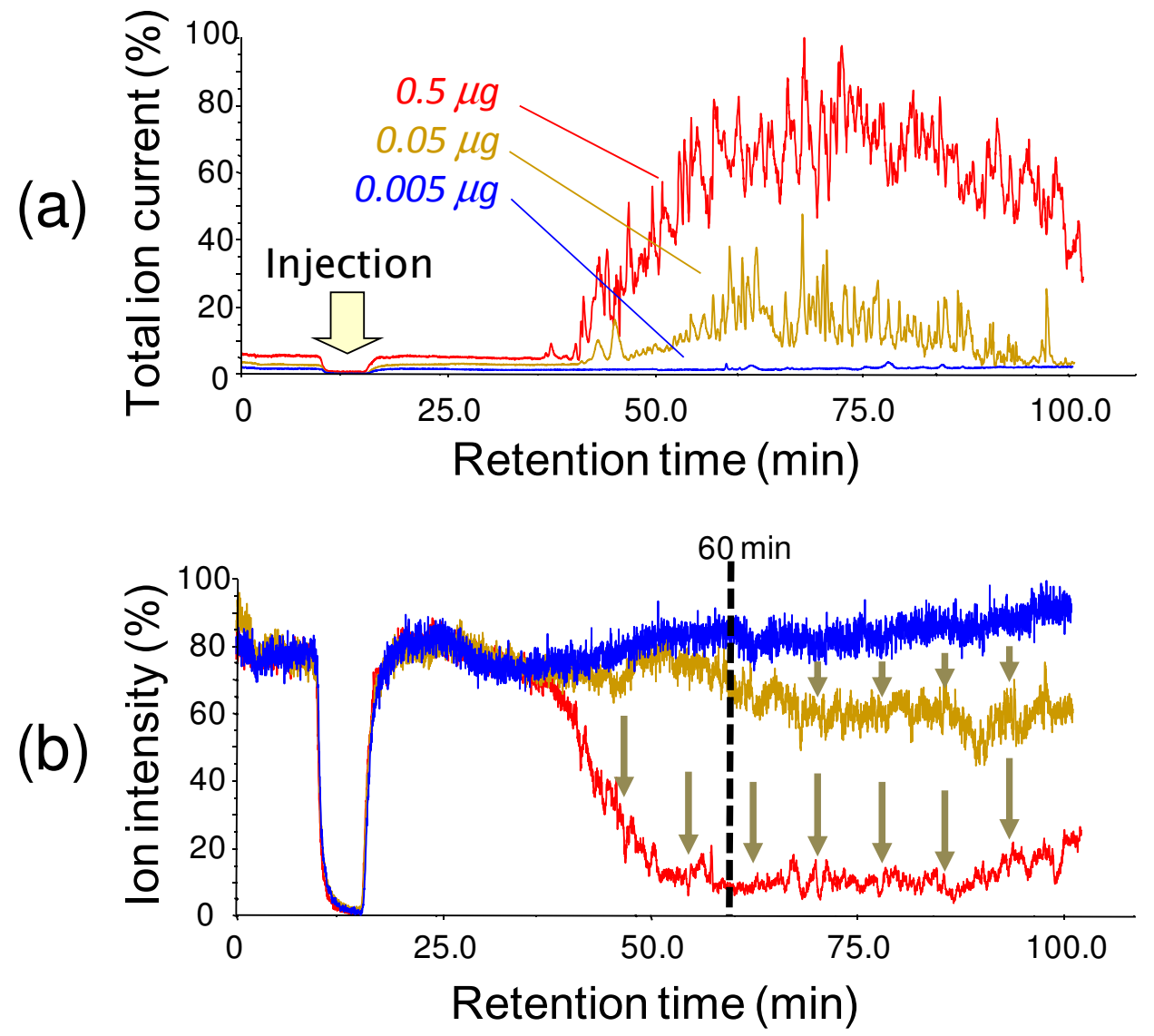

Fig. 5. (a) Total ion current chromatograms obtained from human plasma with injection amounts of $0.005,0.05$, and $0.5 \mu \mathrm{g}$, and (b) corresponding mass chromatograms of the protonated probe.

Figure 5 compares (a) chromatograms of the total ion current and (b) mass chromatograms of the protonated probe. For an injected amount of $0.005 \mu \mathrm{g}$, the mass chromatogram, shown in blue, is rather flat, except for the moment of sample injection using a manual injector. Thus, no ion suppression is likely to occur. However, for $0.05-\mu$ g injection, the intensity for the protonated probe, shown in yellow, decreases appreciably at a retention time above 50 min. For $0.5-\mu$ injection, shown in red, a significant decrease in intensity is detected at a retention time above $40 \mathrm{~min}$. For example, at $60 \mathrm{~min}$ (indicated by the vertical dashed line) the ion intensity for the $0.05-\mu \mathrm{g}$ injection decreases by about $20 \%$, and the decrease is above our experimental error of $10 \%$. This means that potential ion suppression occurs and its maximum effect on ion intensity is $20 \%$. Therefore, if an uncertainty of $20 \%$ is accepted, quantitative analysis is readily performed at the retention time. On the other hand, for the $0.5-\mu \mathrm{g}$ injection, the effect of ion suppression is serious, and quantitative analysis would be difficult. 


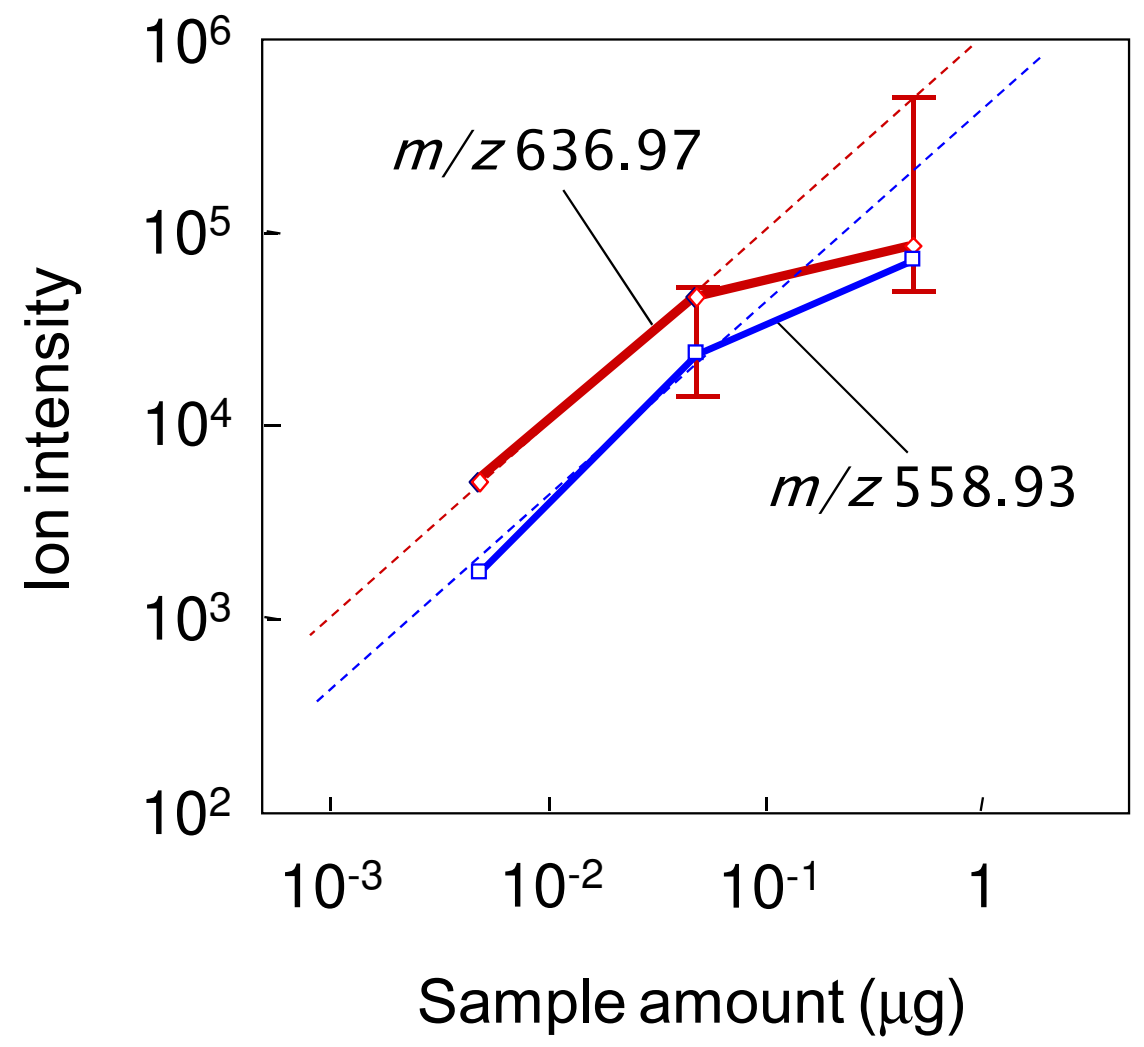

Fig. 6. Peak areas for the $\mathrm{m} / \mathrm{z} 639.91$ and 558.93 ions as a function of the injected sample amount. Broken lines with a slope of 1 are shown as a visual aid. Error bars show the decrease in intensity of the protonated probe.

The probe is expected to be so sensitive to the occurrence of ion suppression that the decrease in intensity for the protonated probe is stronger than those for other protonated molecules. Figure 6 shows the experimental results for protonated molecules chosen randomly. The red line shows the intensity or the peak area for a protonated molecule with $\mathrm{m} / \mathrm{z} 636.97$ detected at a retention time of about $60 \mathrm{~min}$, as a function of the sample injection amount. As the injection amount increases, the difference between the observed intensity and the expected one, shown as a dashed line, increases, and this shows the effect of ion suppression. Another protonated molecule detected at $m / z 558.93$ is also shown in blue. Ion suppression for this ion is detected for the $0.5-\mu \mathrm{g}$ injection, but the decrease in intensity of this ion is weaker than that for the protonated molecule with $\mathrm{m} / \mathrm{z}$ 636.97. Furthermore, the error bars in the figure show the decrease in intensity for the protonated probe at $60 \mathrm{~min}$. The decrease is actually stronger than those for the ion with $\mathrm{m} / \mathrm{z} 636.97$. Therefore, the results are consistent with the above expectation. For the $0.05-\mu \mathrm{g}$ injection, ion suppression may occur for some ions but the intensities of the two ions with $\mathrm{m} / \mathrm{z} 636.97$ and 558.93 are not decreased. 


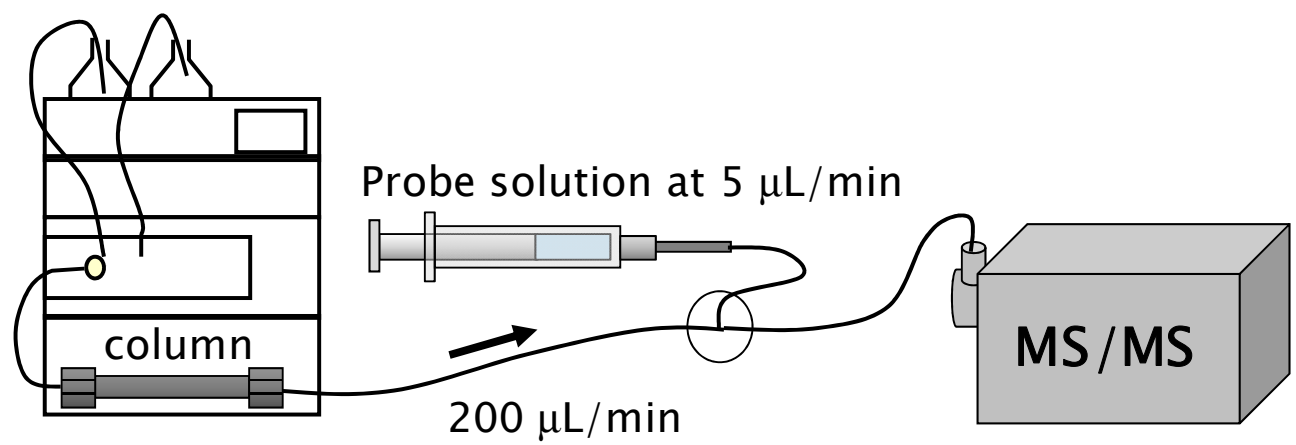

Fig. 7. Experimental setup for LC/MS/MS. An aqueous solution of the probe, pumped by a syringe pump, is mixed with the LC effluent at post-column with a tee.

For semi-micro or conventional LC/MS, the liquid flow rate is so high that the probe solution can be added to the LC mobile phase at post-column through a tee, since the band width of a component separated by the LC column is not expected to be degraded by using a tee with a small dead volume. A typical experimental setup is shown in Figure 7. An aqueous solution of the probe pumped at $5 \mu \mathrm{L} / \mathrm{min}$ is mixed with the LC mobile phase (0.01M- $\mathrm{CH}_{3} \mathrm{COONH}_{4} / \mathrm{CH}_{3} \mathrm{CN} / \mathrm{CH}_{3} \mathrm{COOH}, 600 / 400 / 0.15$; isocratic) in the tee, and the mixed solution is introduced into a triple-quadrupole mass spectrometer operated in selected reaction monitoring (SRM) mode.

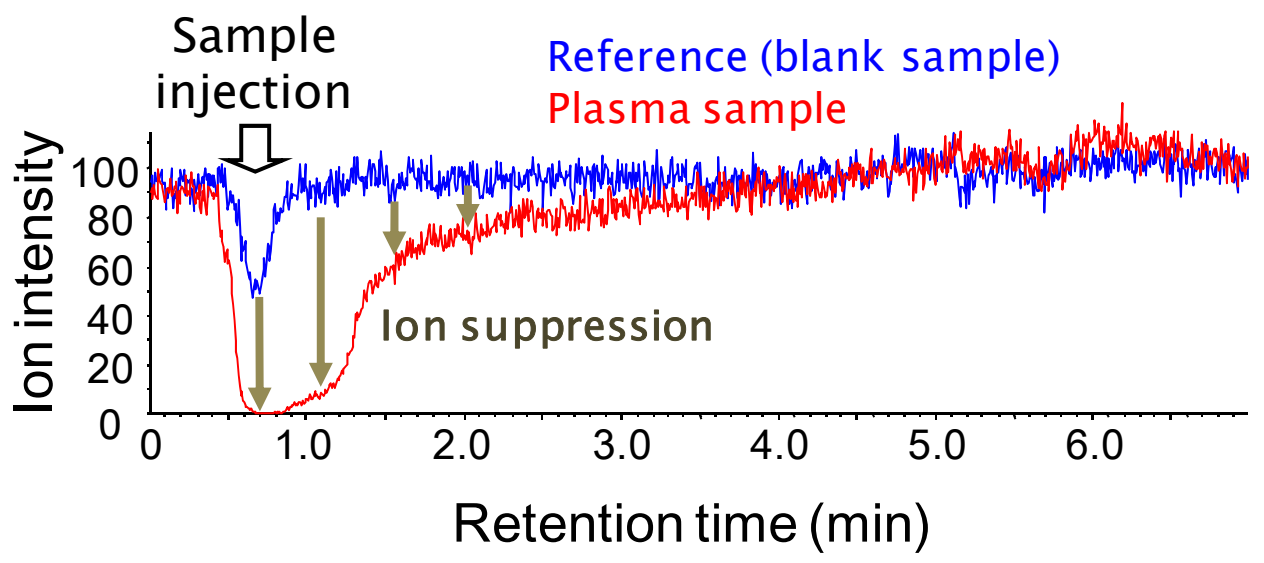

Fig. 8. Mass chromatograms of a fragment ion of the protonated probe for blank and plasma samples.

Before analyzing plasma samples, reference data are obtained using a blank sample such as water. In Figure 8, the mass chromatogram for a fragment $(m / z$ 359.0) of the protonated probe obtained from the blank sample is shown in blue, and that from a plasma sample is shown in red, where the injection amount is about an order of magnitude higher than usual. It is clear that ion suppression is detected just after the sample injection until the retention time of $4 \mathrm{~min}$. In particular, at a retention time of $0.6 \mathrm{~min}$ corresponding to the sample 
injection, the decrease in intensity for the plasma sample is much more significant than that for the reference data. This can be ascribed to an elution of very hydrophilic compounds in the plasma sample.

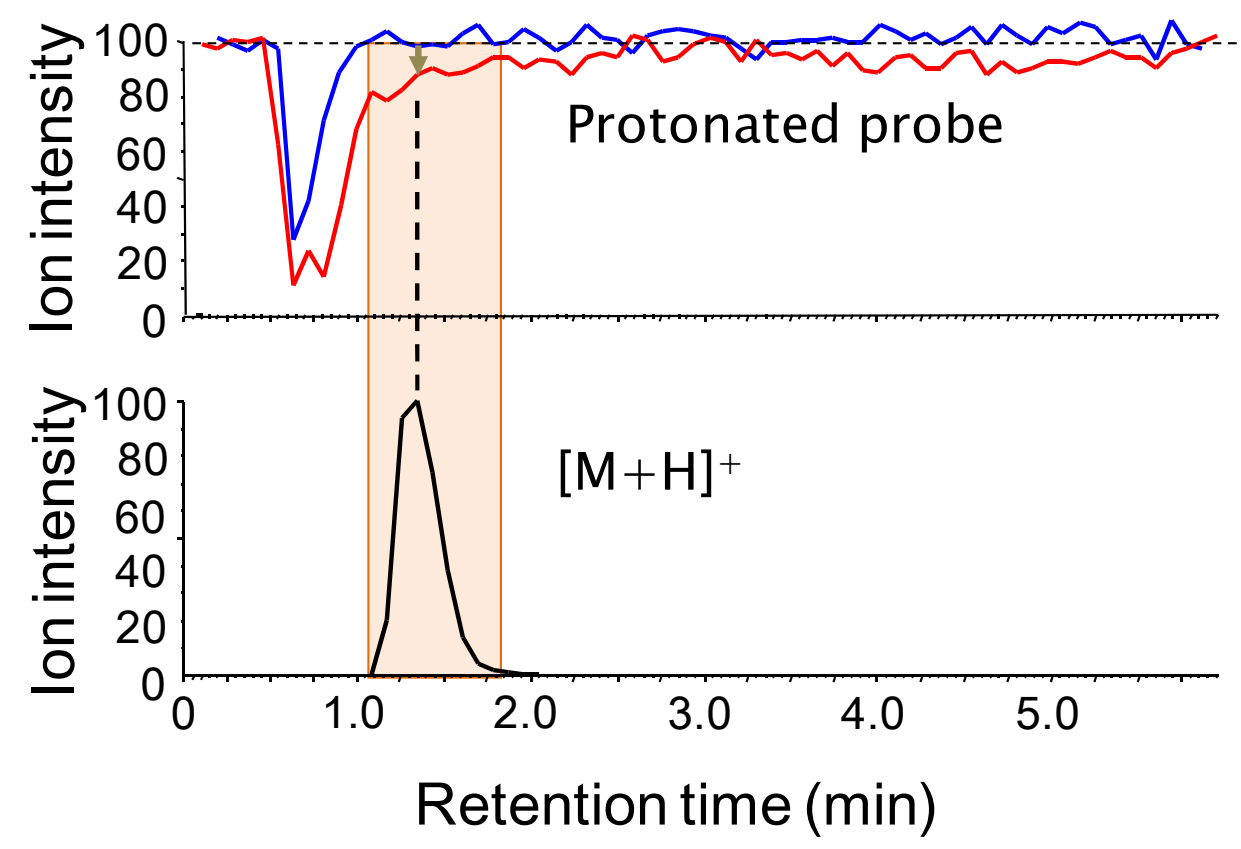

Fig. 9. Mass chromatograms of a fragment ion of the protonated probe for blank and plasma samples, and that of the protonated analyte (Omeprazole).

Figure 9 shows an example of ion-suppression detection obtained under our usual experimental conditions, in which no ion suppression is expected to occur. Omeprazole, a proton-pump inhibitor, is added to human plasma $(4.5 \mu \mathrm{L})$. At a retention time ranging from 1.1 to $1.8 \mathrm{~min}$, the fragment of the protonated Omeprazole is detected at $\mathrm{m} / \mathrm{z} 198.1$, but the intensity of the protonated probe (fragment) at $1.4 \mathrm{~min}$ decreases by $11 \%$, which is beyond our experimental error of about $2 \%$. This means that potential ion suppression occurs, and the intensity of the fragment of the protonated Omeprazole may be suppressed by less than $11 \%$ at 1.4 min since the decrease in the intensity of the protonated probe is expected to be stronger than that of other protonated molecules, as described earlier. On the other hand, the dependence of the peak area (intensity) of the fragment of the protonated Omeprazole on the amount of plasma is shown in Figure 10. The results are obtained under the condition that the injected amount of Omeprazole is constant. The figure shows that the peak area at $4.5 \mu \mathrm{L}$ was $12 \%$ lower than those at 0 and $0.45 \mu \mathrm{L}$. This $12 \%$ decrease in peak area can be ascribed to the decrease in intensity of the protonated probe ranging from 8 to $22 \%$, as shown in Fig. 9 (b). Thus, the decrease in peak area shown in Fig. 10 is almost consistent with the decrease $(11 \%)$ in intensity of the protonated probe (fragment) at the retention time corresponding to the peak intensity for the protonated Omeprazole. Then, if the error of $11 \%$ obtained from the decrease in intensity of the protonated probe at the chromatogram peak is 
acceptable, the data can be readily analyzed quantitatively. If not, we may have to desalt the sample, reduce the injection amount, enhance the fractionation, or modify our LC separation conditions.

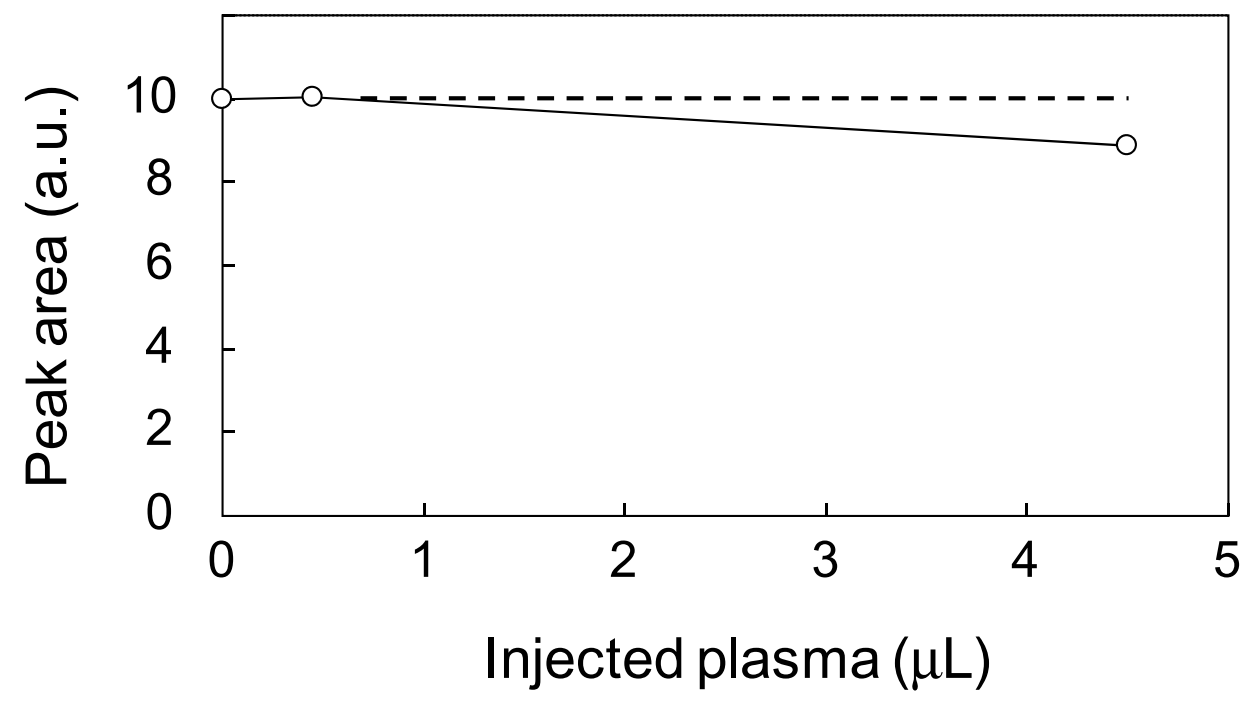

Fig. 10. Peak area (intensity) of the fragment of the protonated Omeprazole as a function of the injected plasma amount.

The detection of ion suppression has been described. As mentioned earlier, ion suppression occurs when the number of charges in the droplet is comparable to, or less than, those of analyte molecules. However, when the number of charges in the droplet increases, ion enhancement occurs. Then, intensities of the protonated molecules increase in accordance with the molecules' chemical properties. Since the increase in charge number can be regarded as a decrease in liquid $\mathrm{pH}$, the protonation for acidic molecules is likely to be enhanced to greater degree than those for neutral and basic ones. This means that intensities of the protonated acidic molecules would increase more than those of other analyte molecules. Thus, when ion enhancement occurs, the intensity of the protonated probe is expected to increase more than those of other analyte molecules. An example of ion enhancement detection is shown in Figure 11. The reference mass chromatogram for the protonated probe, shown in blue, is compared with the mass chromatogram obtained from the plasma sample, shown in red. Ion enhancement is detected at a retention time of about $1.5 \mathrm{~min}$. Furthermore, at 0.5-1.3 min ion suppression is also detected. Because complex matrix effects occur in this case, quantitative analysis of the data is difficult.

The probe can be used for optimizing the sample preparation protocol and analytical conditions as well as for analyzing label-free samples in LC/MS. Furthermore, by monitoring the intensity of the protonated probe, we can detect degradation or pollution of the LC/MS component clearly. Figure 12 compares the mass chromatograms of the protonated probe for the reference data obtained on different days. At about $0.6 \mathrm{~min}$ a decrease in intensity is observed in the green result. This decrease can be ascribed to ion 
suppression caused by contamination at the injection valve. Thus, when the decrease becomes serious, cleaning the valve is recommended.

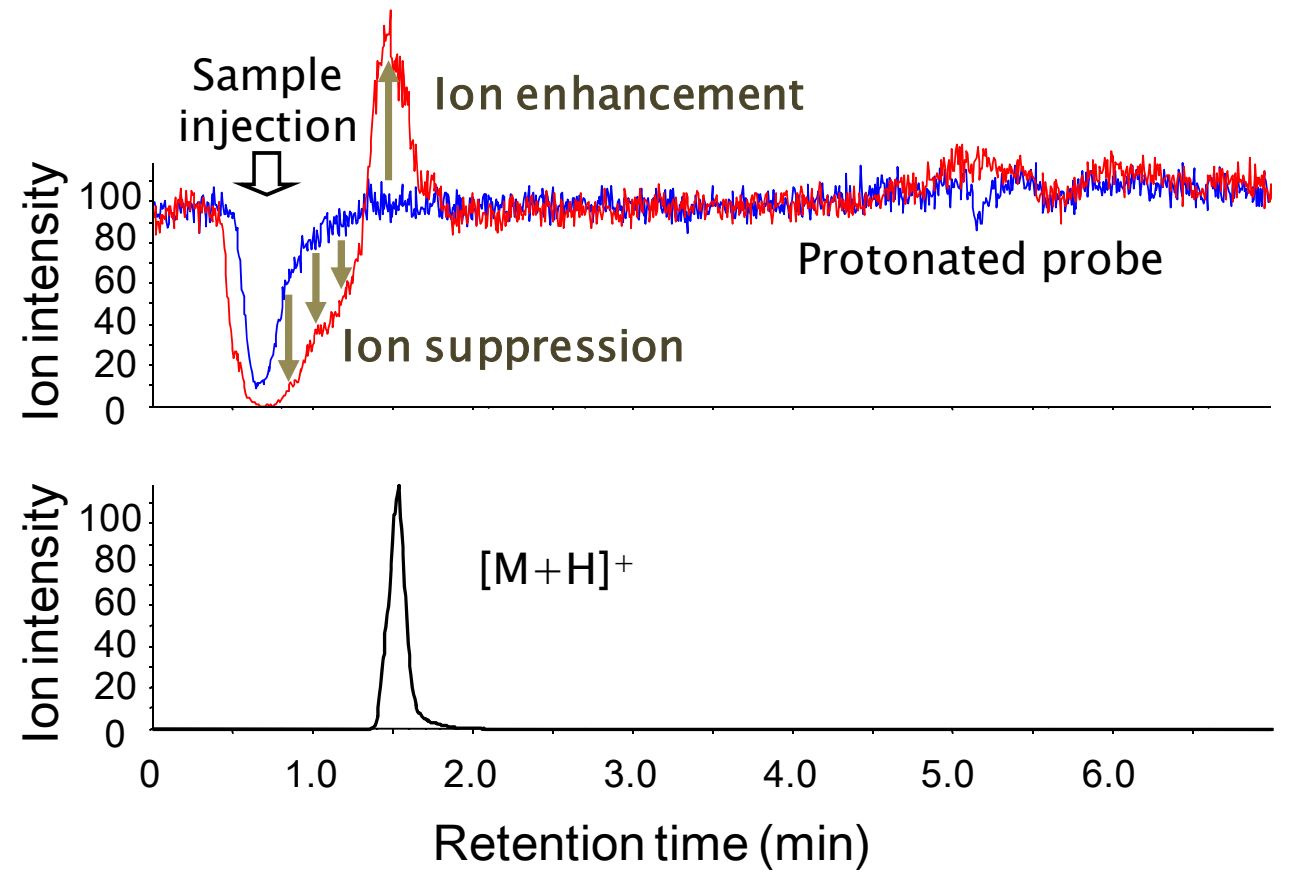

Fig. 11. Typical example of detection of ion enhancement as well as ion suppression. Also, mass chromatogram for a fragment $(\mathrm{m} / \mathrm{z} 294.2)$ of the protonated analyte (Aminopterin) is shown in a lower figure.

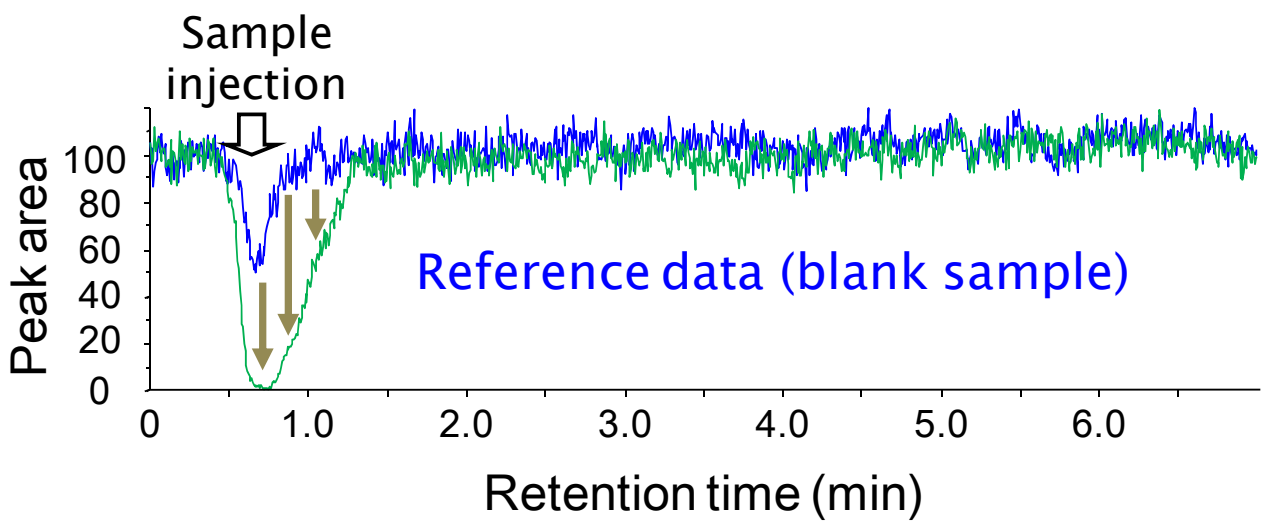

Fig. 12. Comparison of mass chromatograms for a fragment ion of the protonated probe. They were obtained from the blank sample on different days. 
As described above, the potential occurrence of ion suppression and ion enhancement is detected with the probe when the intensity of the protonated probe molecule changes. However, there are some cases where the probe is not always useful for quantitative analysis. Even when neither ion suppression nor ion enhancement is detected with the probe, the intensity of the protonated analyte molecule may possibly have increased. The increase in intensity of the protonated analyte molecule can be ascribed to proton-transfer reactions in the gas-phase with other protonated molecules produced by ESI, not to the matrix effects. Such an increase in intensity occurs only when 1) the analyte such as Aminopterin has a proton affinity much higher than that of the probe and 2) molecules with proton affinities between those of the analyte and the probe are co-eluted with the analyte from LC with high concentrations.

\section{Ion suppression in APCI (Atmospheric Pressure Chemical lonization)}

In APCI, volatile analyte molecules vaporized from an LC effluent by a nebulizer are introduced into a corona discharge plasma, which is generated under atmosphere. In the plasma, protonated solvent molecules such as $\mathrm{H}_{3} \mathrm{O}^{+}$and their hydrated clusters are produced as major reagent ions for chemical ionization in positive ion analysis. In negative ion analysis, on the other hand, anions such as $\mathrm{O}_{2}{ }^{-}$and $\mathrm{OH}^{-}$and their hydrated clusters are major reagent ions. In the plasma, positive and negative charges are balanced. Then, the analyte molecules are ionized by gas-phase ion/molecule reactions such as a proton-transfer reaction and an electron-transfer reaction with the reagent ions in the plasma, as shown below.

$$
\begin{gathered}
\mathrm{H}_{3} \mathrm{O}^{+}+\mathrm{M} \rightarrow \mathrm{H}_{2} \mathrm{O}+[\mathrm{M}+\mathrm{H}]^{+} \text {(proton-transfer reaction) } \\
\mathrm{O}_{2}^{-}+\mathrm{M} \rightarrow \mathrm{O}_{2}+\mathrm{M}^{-} \text {(electron-transfer reaction) }
\end{gathered}
$$

Here, $\mathrm{M}$ is an analyte, and hydrated clusters are omitted for simplicity. The protonated analyte molecule $[\mathrm{M}+\mathrm{H}]^{+}$is produced by the proton-transfer reaction when the analyte has a proton affinity higher than that of $\mathrm{H}_{2} \mathrm{O}$. Thus, the major factor determining the ionization efficiency for the protonated analyte molecule is the proton affinity of the analyte (Hunter\& Lias, 1998). The negatively charged analyte molecule M-, on the other hand, is produced by an electron-transfer reaction when the analyte has an electron affinity higher than that of $\mathrm{O}_{2}$. Thus, the major factor determining the ionization efficiency for the negatively charged analyte molecule is the electron affinity of the analyte (Linde, 1995b). Although the ion/molecule reactions at atmospheric pressure are expected to be almost in equilibrium state, the density of the ion produced by APCI is proportional to that of the reagent ion for the ion/molecule reaction. That means that ion suppression occurs when the density of the reagent ions decreases appreciably. This situation is caused, for example, when the density of co-eluted molecules with proton affinities higher than that of the reagent molecule becomes significant.

In APCI, several kinds of ion/molecule reactions in the gas phase occur such as a protontransfer reaction, electron-transfer reaction, anion-transfer reaction, and anion-attachment reaction (Moini, 2007). Therefore, it is important to identify the reagent ion for the ion/molecule reaction of the analyte molecule before the analysis. Then, the occurrence of ion suppression can be detected by monitoring the intensity of the reagent ion for the 
analyte molecule. Before analyzing biological samples, reference data should be obtained using a blank sample. Potential ion suppression is detected when the intensity of the reagent ion for a biological sample becomes lower than that for the reference sample. In the analysis of protonated analyte molecules, for example, ion suppression can be detected by monitoring the intensity of the protonated solvent molecule such as $\mathrm{H}_{3} \mathrm{O}^{+}$or its hydrated cluster. In the analysis of negatively charged analyte molecules, it can be detected by monitoring the intensity of the anions such as $\mathrm{O}_{2}^{-}$and $\mathrm{OH}^{-}$. In $\mathrm{APCI}$, however, ion suppression occurs less frequently than in ESI probably because charges or the current for the reagent ions, produced in the corona discharge plasma, are much higher than the charges produced by ESI.

\section{Conclusion}

In ESI as an interface in LC/MS, a technique to detect ion suppression or enhancement using a probe has been developed. In positive-ion analysis, the probe should be more hydrophilic and acidic than analyte molecules. In negative-ion analysis, it should be more hydrophilic and basic than analyte molecules. When ion suppression occurs in positive-ion analysis, for example, the intensity of the protonated molecule of the probe is expected to decrease more than those of other analytes. Furthermore, potential error for the intensity of the protonated analyte can be estimated from the decrease in the intensity of the protonated probe.

In preparing a stock solution of the probe, the probe powder is readily dissolved in pure water by adding a small amount of ammonia or trifluoroacetic acid (TFA) to adjust the solution's $\mathrm{pH}$. In an organic-solvent/water solution, however, the very hydrophilic probe might be aggregated in several ten minutes. Thus, the aqueous solution of the probe should be mixed with the LC mobile phase just before the analysis, as shown in Fig. 7.

Directions for the use of the probe are summarized as follows:

1. Add the probe in the LC mobile phase.

2. Obtain reference LC/MS data with a blank sample.

3. Obtain LC/MS data with a biological sample.

4. Compare mass chromatogram for the protonated probe with that in the reference data.

5. Measure potential error from the decrease in intensity for the protonated probe.

The probe can also be used to detect the occurrence of ion enhancement. When ion enhancement occurs, the intensity of the protonated molecule of the probe is expected to increase more than those of other analytes. When neither ion suppression nor ion enhancement occurs, the probe can be used as an internal standard in quantitative LC/MS analysis. Unlike isotope labeling techniques, however, it cannot be used as an internal standard in sample preparation.

In contrast, in APCI the occurrence of ion suppression can be detected by monitoring the intensity of the reagent ion for the analyte.

\section{Acknowledgement}

The author is grateful to M. Furukawa, M. Umeda, T. Bando, Y. Orii, and T. Mori of Hitachi High-Technologies for their help in the experiments and their contribution to our fruitful 
discussions. He also thanks M. Ishimaru, N. Manri, T. Yokosuka, and H. Hanzawa of Hitachi for their invaluable assistance.

\section{References}

Tang, K.; Page, J. \& Smith, R. (2004). Charge Competition and the Linear Dynamic Range of Detection in Electrospray Ionization Mass Spectrometry, Journal of the American Society for Mass Spectrometry, Vol. 15, Issue 10, (April 2004), pp. 1416-1423, ISSN 1044-0305

Buhrman, D.; Price, P. \& Rudewicz, P. (1996). Quantitation of SR 27417 in Human Plasma using Electrospray Liquid Chromatography-Tandem Mass Spectrometry: A Study of Ion Suppression, Journal of the American Society for Mass Spectrometry, Vol. 7, Issue 11, (June 1996), pp. 1099-1105, ISSN 1044-0305

Matuszewski, B.; Constanzer, M. \& Chevez-Eng, C. (1998). Matrix Effect in Quantitative LC/MS/MS Analyses of Biological Fluids: A Method for Determination of Finasteride in Human Plasma at Picogram Per Milliliter Concentrations. Analytical Chemistry, Vol. 70, Issue 5, (December 1997), pp. 882-889, ISSN 1520-6882

Bonfiglio, R.; King, R.; Olah, T. \& Merkle, K. (1999). The Effects of Sample Preparation Method on the Variability of the Electrospray Ionization Response for Model Drug Compounds. Rapid Communications in Mass Spectrometry, Vol. 13, Issue 12, (April 1999), pp. 1175-1185, ISSN 1097-0231

Hirabayashi, A.; Ishimaru, M.; Manri, N.; Yokosuka, T. \& Hanzawa, H. (2007). Detection of Potential Ion Suppression for Peptide Analysis in Nanoflow Liquid Chromatography/Mass Spectrometry, Rapid Communications in Mass Spectrometry, Vol. 21, Issue 17, (June 2007), pp. 2860-2866, ISSN 1097-0231

Hirabayashi, A.; Furukawa, M.; Umeda, M.; Bando, T. \& Orii, Y. (2009). Probe for Label-free Quantitative Analysis in Liquid Chromatography/Mass Spectrometry, Analytical Sciences, Vol. 25, Number 1, (January 2009), pp. 67-71, ISSN 0910-6340

Iribarne, J. \& Thomson, B. (1976). On the Evaporation of Small Ions from Charged Droplets, Journal of Chemical Physics, Vol. 64, Number 6, (July 1975), pp. 2287-2294, ISSN 00219606

de la Mora, J. (2000). Electrospray Ionization of Large Multiply Charged Species Proceeds via Dole's Charged Residue Mechanism, Analytica Chimica Acta, Vol. 406, Issue 1, (February 1999), pp. 93-104, ISSN 0003-2670

Dole, M.; Mack, L.; Hines, R.; Mobley, R.; Ferguson, L. \& Alice, M. (1968). Molecular Beams of Macroions, Journal of Chemical Physics, Vol. 49, Number 5, (April 1968), pp. 22402249, ISSN 0021-9606

Hirabayashi, A.; Takada, Y.; Kambara, H.; Umemura, Y.; Ito, H. \& Kuchitsu, K. (1993). Mass Spectroscopic Studies of Protonation to Amino-acid Molecules in Atmospheric Pressure Spray, Chemical Physics Letters, Vol. 204, Number 1,2, (December 1992), pp. 152-156, ISSN 0009-2614

Black, S. \& Mould, D. (1991). Development of Hydrophobicity Parameters to Analyze Proteins Which Bear Post- or Cotranslational Modifications, Analytical Biochemistry, Vol. 193, Issue 1, (July 1990), pp. 72-82, ISSN 0003-2697

Linde, D. (1995a). Section 7, Biochemistry, In: CRC Handbook of Chemistry and Physics, pp. 1, CRC Press, ISBN 0-8493-0476-8, Boca Raton 
Gasteiger, E.; Hoogland, C.; Gattiker, A.; Duvaud, S.; Wilkins, M.; Appel, R. \& Bairoch, A. (2005). Protein Identification and Analysis Tools on the ExPASy Server, In: The Proteomics Protocols Handbook, J.M. Walker (Ed), pp. 571-607, Humana Press, ISBN 798-1-58829-383-5, Available from http://au.expasy.org/tools/pi_tool.html

Moini, M. (2007). Atmospheric Pressure Chemical Ionization: Principles, Instrumentation, and Applications, In: The Encyclopedia of Mass Spectrometry Volume 6; Ionization Methods, M. Gross \& R. Caprioli, (Ed.), pp. 345-354, ISBN 978-0-08-0438016, Elsevier, Amsterdam

Hunter, E. \& Lias, S. (1998). Evaluated Gas Phase Basicities and Proton Affinities of Molecules: An Update. Journal of Physical and Chemical Reference Data, Vol. 27, Issue 3, (August 1997), pp. 413-656, ISSN 0047-2689

Linde, D. (1995b). Section 10, Atomic, Molecular, and Optical Physics, In: CRC Handbook of Chemistry and Physics, pp. 180-188, CRC Press, ISBN 0-8493-0476-8, Boca Raton 


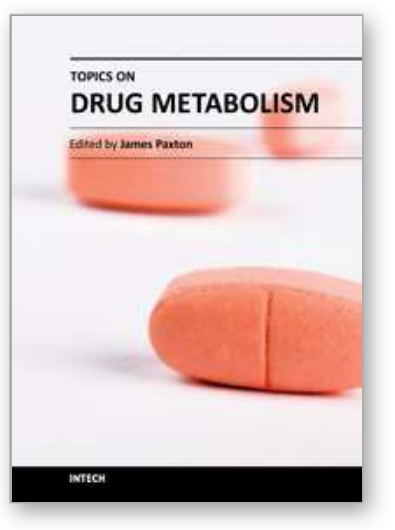

\author{
Topics on Drug Metabolism \\ Edited by Dr. James Paxton
}

ISBN 978-953-51-0099-7

Hard cover, 294 pages

Publisher InTech

Published online 22, February, 2012

Published in print edition February, 2012

In order to avoid late-stage drug failure due to factors such as undesirable metabolic instability, toxic metabolites, drug-drug interactions, and polymorphic metabolism, an enormous amount of effort has been expended by both the pharmaceutical industry and academia towards developing more powerful techniques and screening assays to identify the metabolic profiles and enzymes involved in drug metabolism. This book presents some in-depth reviews of selected topics in drug metabolism. Among the key topics covered are: the interplay between drug transport and metabolism in oral bioavailability; the influence of genetic and epigenetic factors on drug metabolism; impact of disease on transport and metabolism; and the use of novel microdosing techniques and novel LC/MS and genomic technologies to predict the metabolic parameters and profiles of potential new drug candidates.

\title{
How to reference
}

In order to correctly reference this scholarly work, feel free to copy and paste the following:

Atsumu Hirabayashi (2012). Label-Free Quantitative Analysis Using LC/MS, Topics on Drug Metabolism, Dr. James Paxton (Ed.), ISBN: 978-953-51-0099-7, InTech, Available from:

http://www.intechopen.com/books/topics-on-drug-metabolism/label-free-quantitative-analysis-using-lc-ms

\section{INTECH}

open science | open minds

\section{InTech Europe}

University Campus STeP Ri

Slavka Krautzeka 83/A

51000 Rijeka, Croatia

Phone: +385 (51) 770447

Fax: +385 (51) 686166

www.intechopen.com

\section{InTech China}

Unit 405, Office Block, Hotel Equatorial Shanghai

No.65, Yan An Road (West), Shanghai, 200040, China

中国上海市延安西路65号上海国际贵都大饭店办公楼 405 单元

Phone: +86-21-62489820

Fax: $+86-21-62489821$ 
(C) 2012 The Author(s). Licensee IntechOpen. This is an open access article distributed under the terms of the Creative Commons Attribution 3.0 License, which permits unrestricted use, distribution, and reproduction in any medium, provided the original work is properly cited. 